\title{
Entrelacs
}

Cinéma et audiovisuel

Hors-série $n^{\circ} 4$ | 2016

Paysages en séries

\section{L'homme contre la nature dans True Detective}

L'homme contre la nature dans True Detective

\section{Marie Maillos}

\section{(2) OpenEdition}

Journals

Édition électronique

URL : http://journals.openedition.org/entrelacs/2137

DOI : 10.4000/entrelacs.2137

ISBN : $2261-5482$

ISSN : 2261-5482

Éditeur

Éditions Téraèdre

Édition imprimée

Date de publication : 1 novembre 2016

ISSN : 1266-7188

Référence électronique

Marie Maillos, «L'homme contre la nature dans True Detective », Entrelacs [En ligne], Hors-série n

4 | 2016, mis en ligne le 06 février 2018, consulté le 04 mai 2019. URL : http://

journals.openedition.org/entrelacs/2137; DOI : 10.4000/entrelacs.2137

Ce document a été généré automatiquement le 4 mai 2019.

Tous droits réservés 


\title{
L'homme contre la nature dans True Detective
}

\author{
L'homme contre la nature dans True Detective
}

Marie Maillos

1 Dans la première saison de la série de Nic Pizzolatto True Detective, la nature de Louisiane, particulièrement présente et remarquable, offre des paysages dont l'aspect sauvage n'est pas sans faire penser aux jungles devenues mythiques du Vietnam - lesquelles servaient d'ennemi parfois plus puissant que l'ennemi lui-même aux soldats américains - telles que représentées dans de nombreux films depuis. C'est que cette nature est dans la série un adversaire : non pas un adversaire à toute forme d'humanité, puisque de jeunes prostitués par exemple y trouvent un abri loin de la violence des hommes, mais un adversaire à la virilité. Ainsi, dans la première saison de True Detective, tout homme est un soldat qui met sa virilité en jeu contre la Nature hostile. Les deux soubassements de la construction de la virilité - la famille et la guerre - deviennent donc des questions d'ordre naturel, dont le paysage et ses lignes nous disent quelque chose.

\section{L'ordre naturel}

Dans un premier temps, True Detective présente un ordre "naturel», celui du rapport triangulaire de l'homme, de la femme et de l'enfant, dans lequel l'homme est un élément déterminant mais instable. L'évidence naturelle de la famille est soulignée dès le premier épisode par Martin Hart, qui l'a a priori parfaitement intégrée, lorsqu'il dit « Past a certain age a man without a family can be a bad thing ${ }^{1}$ " (épisode pilote). Dans son univers, tout indique d'ailleurs qu'il ait raison. Les familles en Louisiane s'étendent infiniment, leur patronyme étant gage de cette conquête : on retrouve les noms de Lange, Fontenot, Childress ou Tuttle sur différentes générations. Martin le souligne encore lorsqu'il déclare aux enquêteurs "I wanted every member of that family that I could find. There weren't any. You know how weird that is in Louisiana ${ }^{2}$ ?» (Épisode 4). Par ailleurs les personnages féminins qui gravitent - toujours dans des rôles secondaires - autour des personnages principaux sont définis par leur rôle dans la logique jugée « naturelle » de la procréation: 
Maggie est une mère, les deux filles de Martin sont les filles, et les prostituées sont sans cesse à la recherche d'un foyer (c'est le cas de la première amante de Marty, qui est à la recherche d'un « homme bien » avec qui fonder une famille) ou d'un père de substitution - comme c'est le cas de la jeune prostituée que Martin aide financièrement lorsqu'elle lui semble être une petite fille, mais qui devient son amante lorsqu'elle devient une femme.

Dans l'univers de Rustin Cohle, au contraire, il n'y a pas d'« ordre » naturel, et la famille ou la procréation sont considérées comme des erreurs de la Nature. Il affirme d'abord à son collègue "we are creatures that should not exist by natural law " puis "I think the honorable thing for our species to do is to deny our programming, stop reproducing, walk hand in hand into extinction ${ }^{4}$. (Épisode 1). Plus tard il commentera à nouveau cette idée avec les deux enquêteurs en disant de sa fille défunte "she spared me the sin of being a father ${ }^{5}$ " (épisode 2).

4 C'est dans la confrontation de ces deux conceptions de la famille que Martin Hart et Rustin Cohle se définissent l'un par rapport à l'autre. Ils font ainsi le procès permanent de leur propre virilité, tandis que les enfants, dans la série, apparaissent aussi bien en tant que victimes qu'en tant que juges de leurs « crimes ».

\section{Prédateurs et protecteurs}

5 Dans cet ordre naturel controversé, leur virilité fait tantôt des deux détectives des prédateurs, ce que Rustin Cohle assume tout à fait, tantôt des protecteurs, ce vers quoi tend Martin Hart sans jamais y parvenir pleinement. Leur violence, toujours surgie d'une volonté protectrice, s'avère chaque fois destructrice et dangereuse : c'est le cas lorsque Martin Hart tue dune balle dans la tête Reggie Ledoux, responsable de la mort des enfants, et c'est le cas encore lorsqu'il passe à tabac les amants de sa fille pour " protéger » celle-ci. Alors que Martin est déstabilisé par sa propre violence qui le ferait facilement passer du statut de protecteur à celui de prédateur, Rustin Cohle l'embrasse en assumant le fait d'être un homme "mauvais", mais en affirmant : "The world needs bad men. We keep the other bad men from the door ${ }^{6}$. " Il réconcilie ainsi les deux éléments du paradoxe qui déchire Martin Hart. Et en effet la figure procréatrice dans la série est celle de la femme, tournée vers l'avenir et l'enfantement, tandis que l'homme reste une figure destructrice et stérile. Cet aspect destructeur est représenté aussi bien par la violence des personnages masculins que par les diverses références qui sont faites à la guerre de Corée et du Vietnam, au travers notamment de l'histoire de leurs pères respectifs, tous deux vétérans. Martin et Rustin rejoueront d'ailleurs cette guerre lorsqu'ils iront appréhender - puis tuer - le dealer de méthamphétamine. La nature abondante leur servira alors de décor presque cliché de la guerre du Vietnam, alors qu'ils apprendront comme leurs pères - et grâce aux leçons de chasse de celui de Rustin Cohle - à tirer partie des pièges de leurs opposants et des éléments naturels pour semer la mort et la destruction, de façon violente, explosive, et moralement ambiguë. 


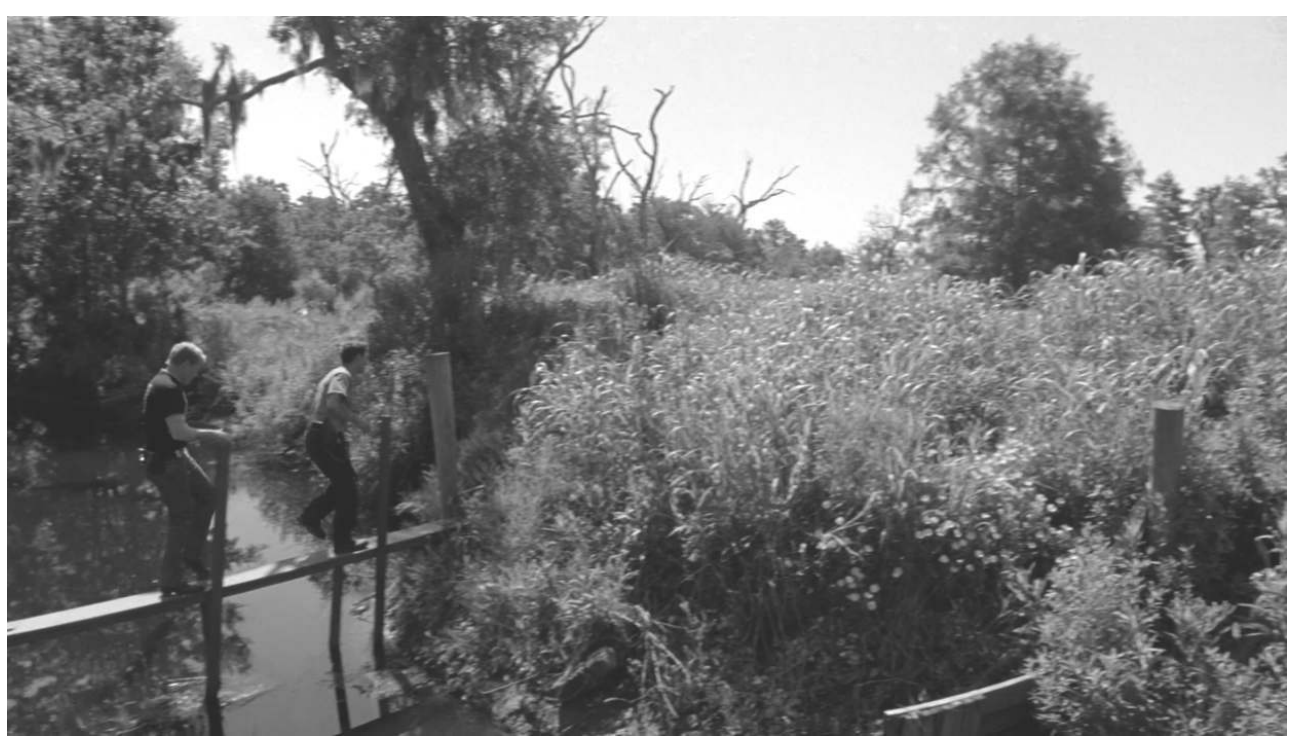

Fig 1 : Les personnages tirent partie des pièges de la nature

6 C'est en outre cette image de la guerre du Vietnam, dans laquelle les hommes se battent autant contre l'ennemi que contre la Nature, que l'on retrouvera dans le dernier épisode de la saison:

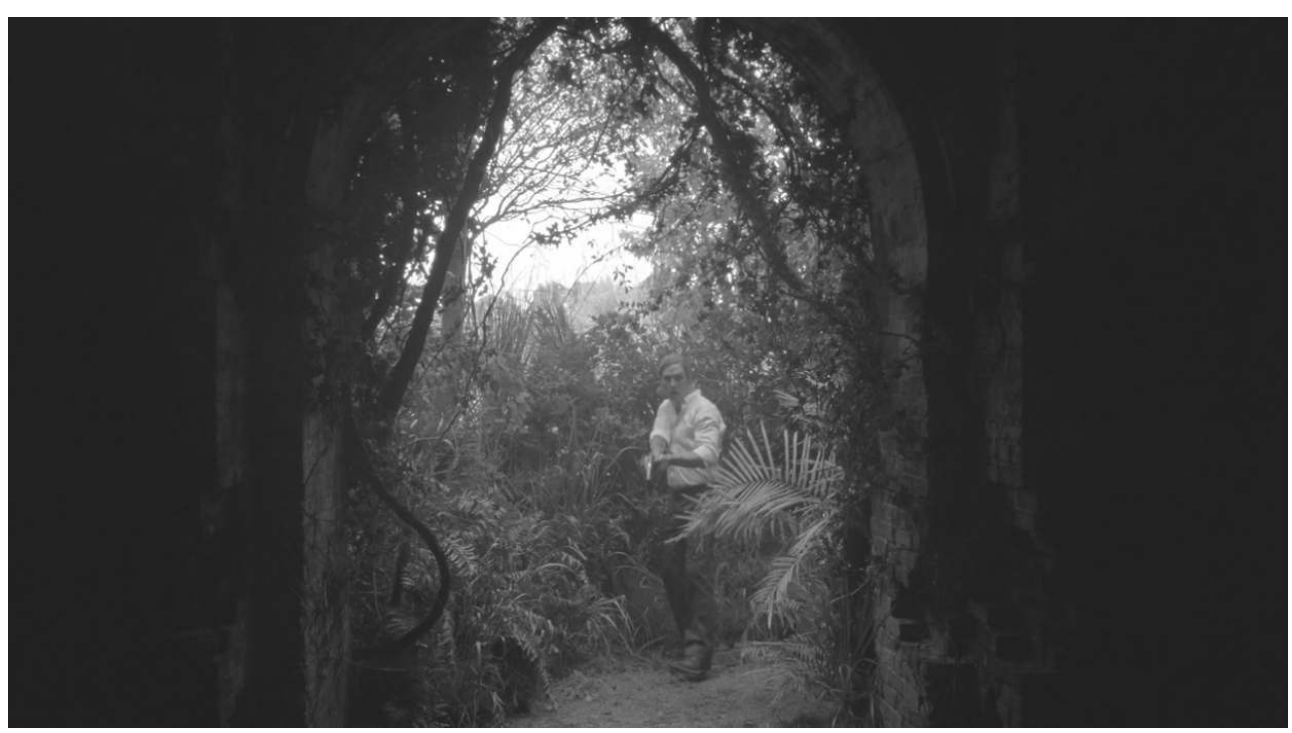

Fig 2 : Rustin Cohle rejoue la Guerre du Viêt-Nam

7 Ils s'incluent donc tous deux dans une généalogie patriarcale entièrement tournée vers la destruction. A contrario, il n'est fait référence qu'une seule fois à la mère de l'un d'eux, celle de Martin Hart, et c'est en tant que «Donna Reed type ${ }^{7}$ ", femme-mère aimante et prévenante, modèle auquel Martin Hart essaiera de conformer sa femme, mais jamais luimême. Il est d'ailleurs intéressant de souligner que la véritable Donna Reed fut mariée quelques années au maquilleur William Tuttle, dont le nom traverse la série comme étant celui de la figure paternelle absolue : homme de pouvoir, fondateur d'un certain nombre d'écoles (ce qui fera de lui le père de tous les enfants qui s'y trouvent) ... et responsable de la disparition, de la torture et de la mort d'un certain nombre d'entre eux. Ce couple archétypal de la bonne mère Donna Reed et du mauvais père Tuttle pèse donc sur les personnages principaux. 
Car les personnages masculins, au-delà de leur propension à la destruction, sont aussi caractérisés par une forme de stérilité. Lorsque Rustin Cohle raconte son enfance en Alaska, c'est le désert aride et froid qu'il met en valeur. Mais lorsqu'il quitte son père - et l'Alaska - c'est pour se rendre dans le désert aride et brûlant du Texas. Ces deux États gigantesques et plats sont la représentation même de sa virilité. Cette forme de stérilité, Rustin Cohle est forcé de l'assumer puisqu'il a perdu quelques années auparavant sa fille. Martin Hart, par contre, refuse de la reconnaître, alors qu'il perd petit à petit la communication avec ses filles. De fait, lorsqu'ils exécutent deux criminels et récupèrent sur place des enfants martyrisés, Rustin porte le garçon, mort depuis deux jours déjà, tandis que Martin tient la fille, encore vivante mais plongée à jamais dans un état de catatonie qui l'empêchera justement de communiquer avec qui que ce soit.

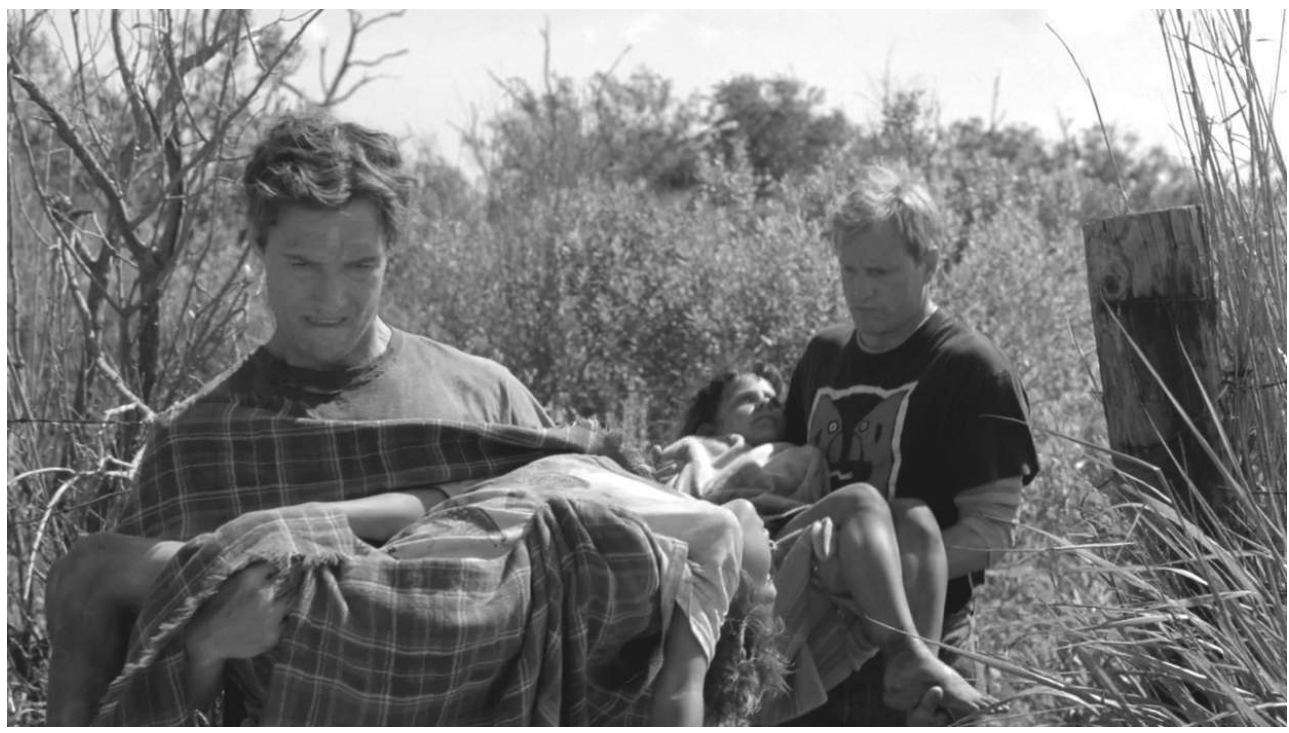

Fig 3 : Rustin Cohle porte le garçon décédé et Martin Hart la fille apathique

9 Or cette stérilité du masculin est montrée dans le film comme un aspect non-naturel : la Nature est liée à la féminité, tandis que l'homme masculin est cette créature « that should not exist by natural law» dont parle Cohle. Il y a donc perpétuellement un effet de «dénaturation» des personnages masculins de la série. La figure de l'oncle de Marie Fontenot en est l'indice : cet homme, qui devrait être "parfaitement viril » puisqu'il est un sportif professionnel et que Martin lui-même l'érige en héros de son enfance, n'est plus capable de bouger ni même de parler correctement lorsque Martin et Rustin viennent lui rendre visite: il a été proprement dénaturé par une série d'attaques cérébrales. L'alcool pervertit de la même façon d'autres personnages masculins: l'alcoolisme de Marty le rend violent et jaloux, celui de Rustin finit par déformer son visage, celui du prêcheur lui fait perdre la Foi, et ainsi de suite. Quand la dénaturation des hommes n'est pas organique, elle est mécanique, tout en gardant cet aspect monstrueux de protubérance, de déviation du naturel : la drogue par exemple transforme le cuisinier de méthamphétamine en ce monstre dont le visage a été remplacé par un masque lui donnant une allure hybride, mi-homme, mi-machine. 


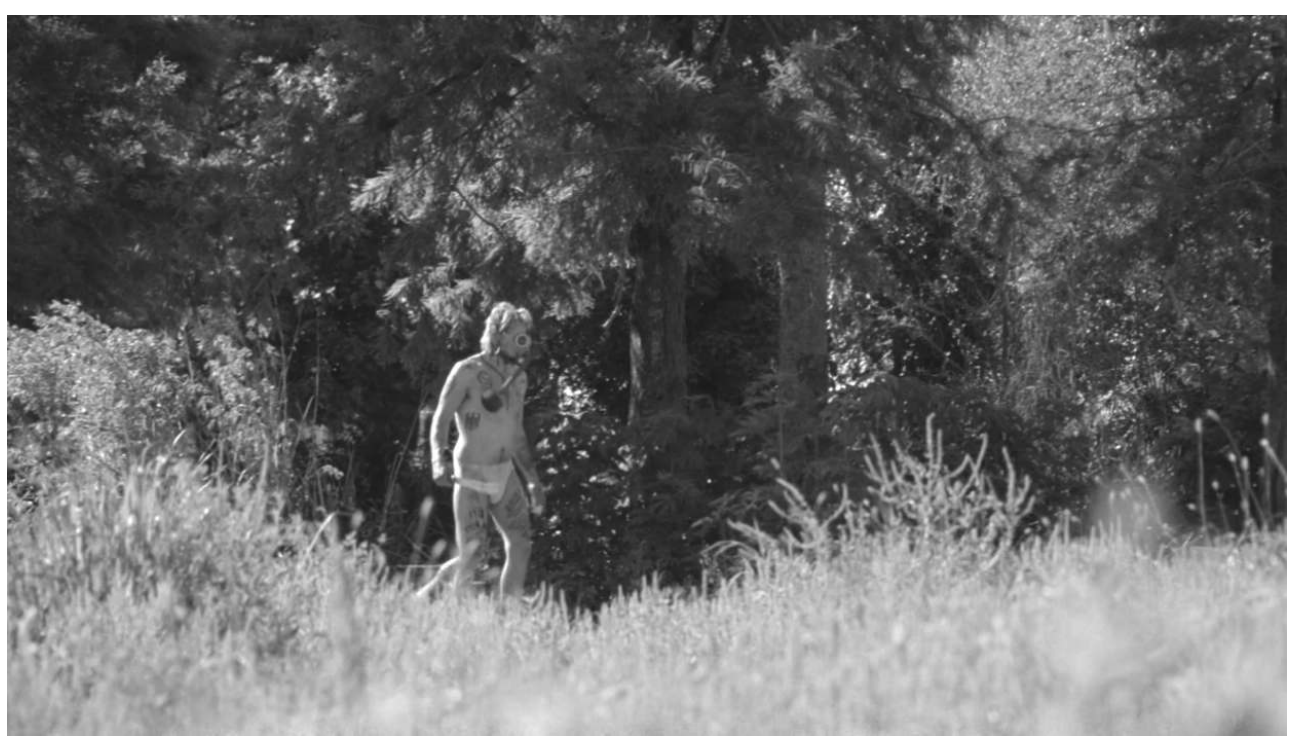

Fig 4 : Mi-homme, mi-machine

10 La voiture elle-même est utilisée par tous les hommes du film comme un prolongement mécanique de leur être, qui les hybride chaque fois et les éloigne de leur état naturel. Aucune voiture n'est d'ailleurs conduite par une femme dans cette saison de la série.

\section{Une guerre par lignes}

11 Cette guerre intestine de l'homme contre la Nature est parfaitement représentée visuellement par la guerre des lignes et par les paysages. La composition des plans de True Detective est entièrement tournée vers la confrontation des lignes courbes et des lignes droites. Les lignes courbes, souvent tortueuses, sont celles des arbres, de la nature, des femmes au corps plein dont les seins sont souvent dévoilés, etc. Au contraire les lignes droites sont celles des paysages arides, des deux héros aux visages taillés à la serpe et émaciés. Cette confrontation géométrique se retrouve d'ailleurs dans la guerre symbolique qui s'effectue dans la série entre la religion catholique représentée par la croix, et les rites païens et vaudous représentés par la spirale. Les hommes sont cette croix : leurs œuvres industrielles maintenant inanimées sont de longues lignes verticales, à leur image d'« homo-erectus », qui viennent briser les paysages aux lignes horizontales souvent mises en valeur par les nombreux plans en hélicoptère, comme c'est déjà le cas à la cinquième minute de l'épisode pilote. Le scénariste tire ainsi parti du territoire particulier de la Louisiane, dont le point culminant ${ }^{88}$ ne s'élève qu'à cent soixante trois mètres. 


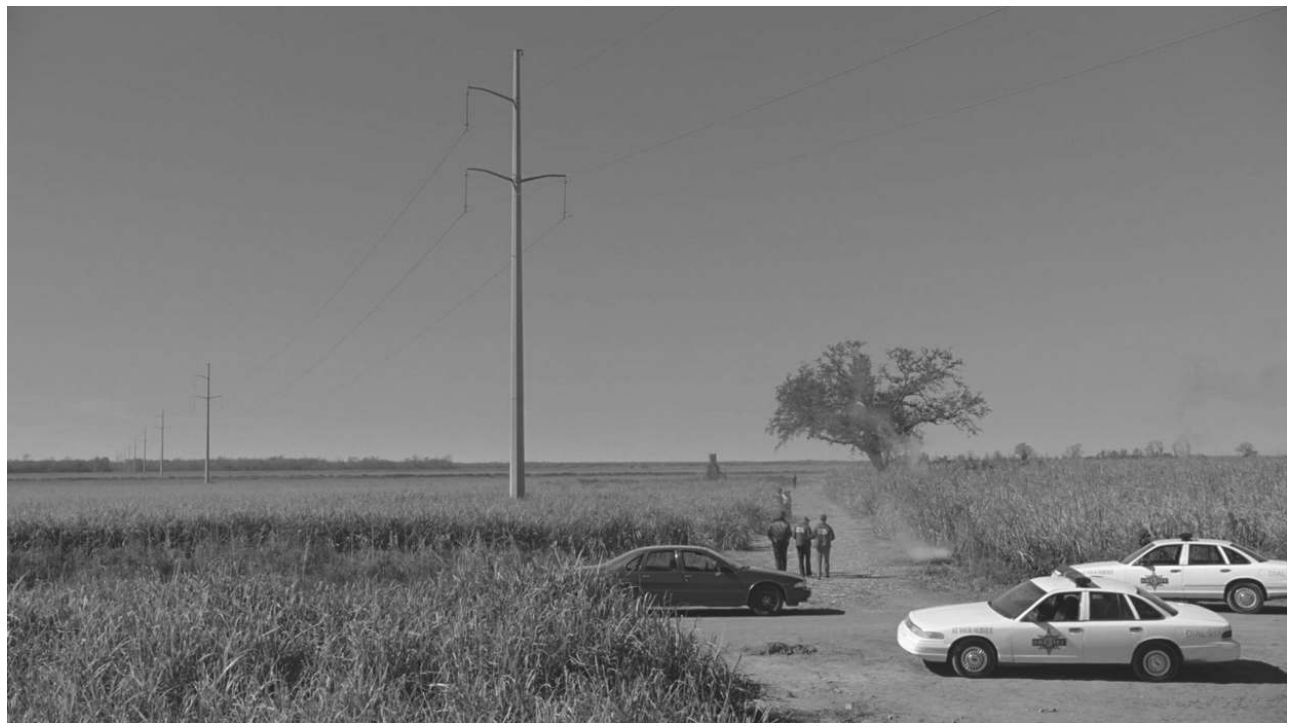

Fig 5 : Lignes droites, épisode pilote

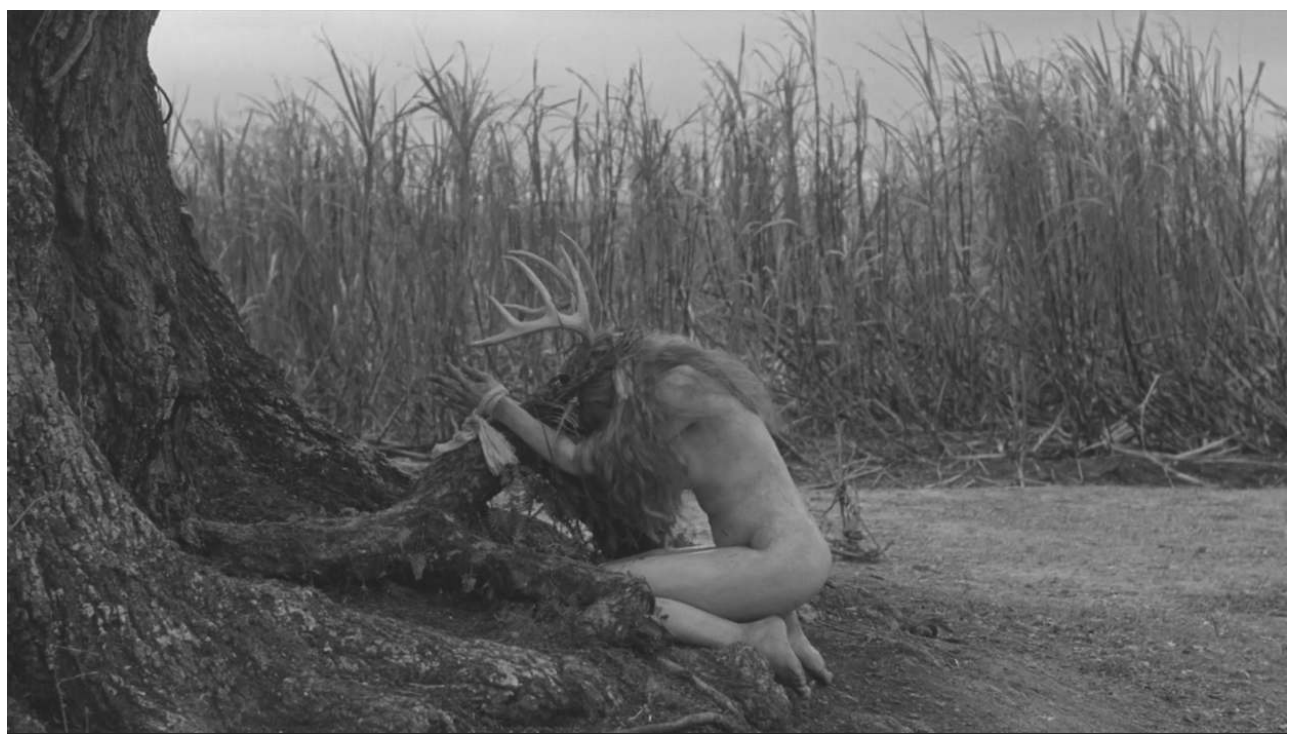

Fig 6 : Lignes courbes, épisode pilote

12 Ces lignes sont aussi celles que l'on retrouve dans l'appartement de Rustin Cohle, particulièrement lorsque Martin y entre pour la première fois : le lit à même le sol et l'absence de décoration, si ce n'est la croix qui vient faire écho à l'ensemble, crée encore une fois une composition à deux lignes droites, perpendiculaires. 


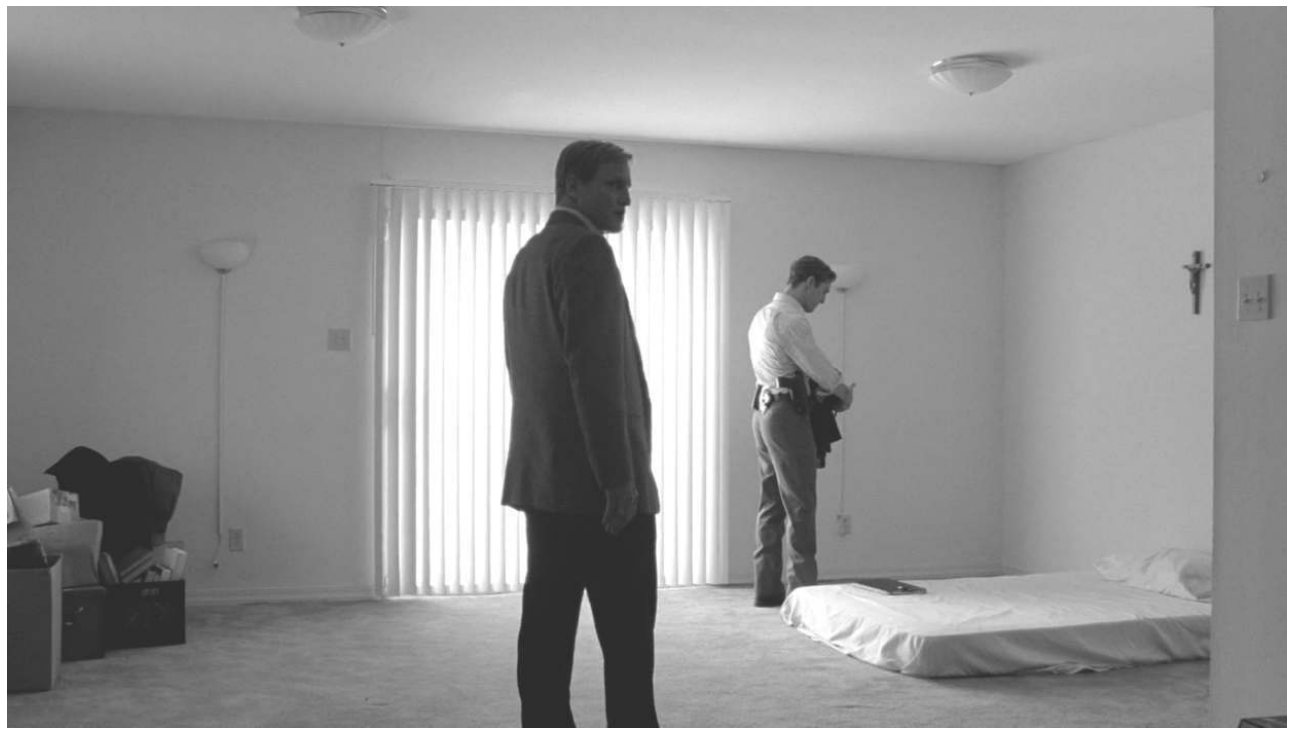

Fig 7 : L'appartement de Rustin Cohle, épisode pilote

13 L'arbre et la spirale, au contraire, menacent chaque fois ces espaces - géométriques et donc hors de la Nature - d'une destruction organique. Les plans de deux églises, dans les épisodes 1 et 2 , en sont un exemple expressif: la nature ronge le plan, laissant voir les lignes droites et discontinues de ces églises.

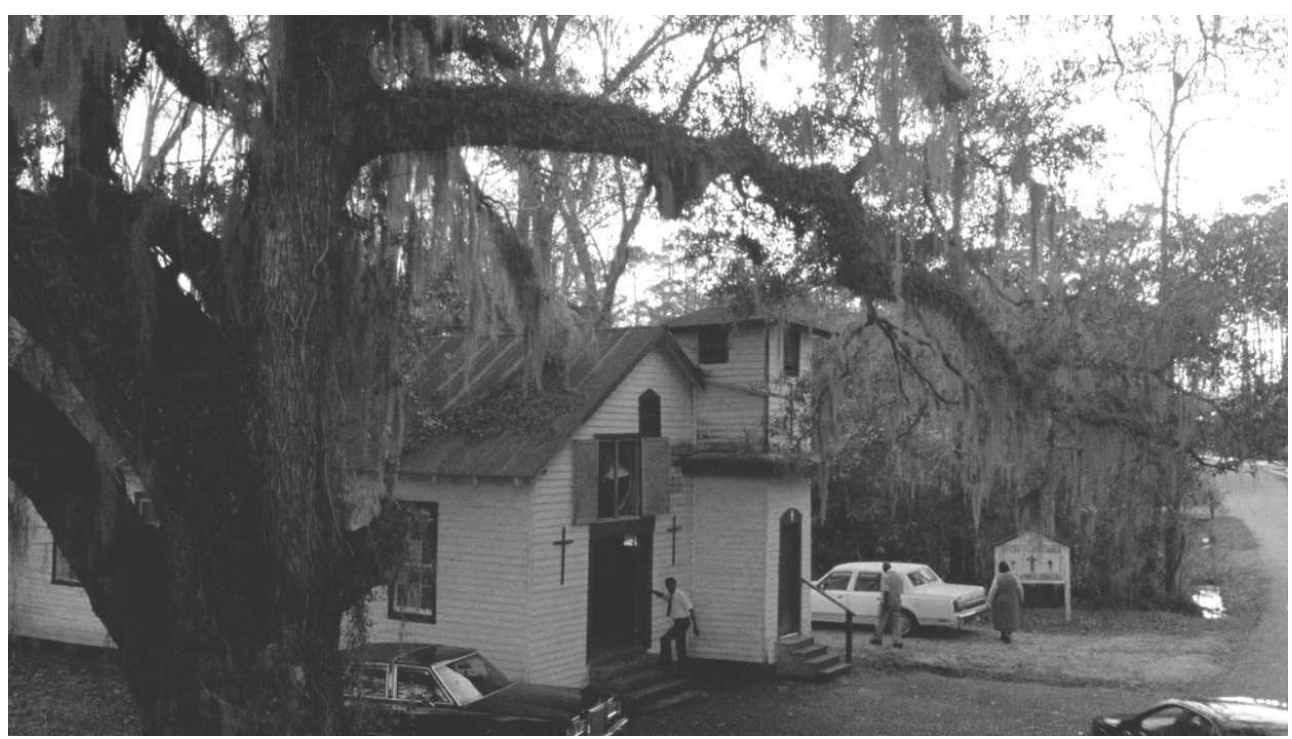

Fig 8 : Église, épisode pilote 


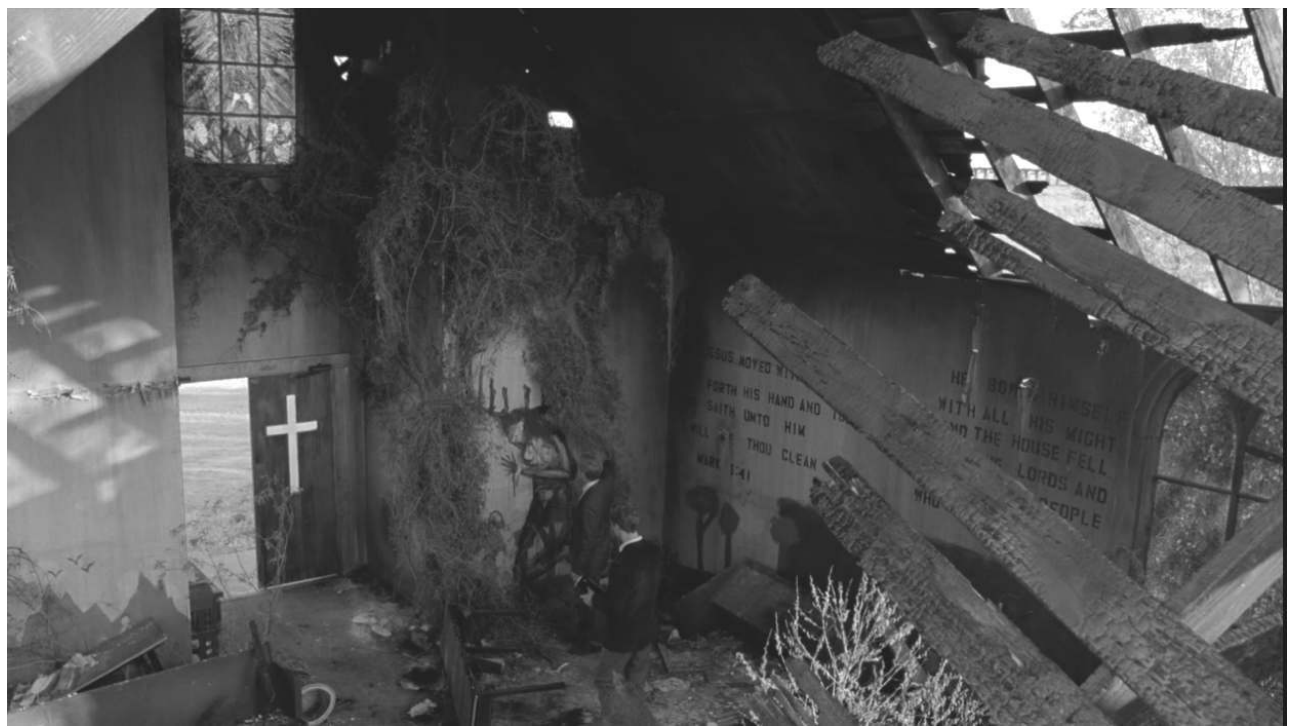

Fig 9 : Église, deuxième épisode

14 Ce sont d'ailleurs ces mêmes lignes droites et courbes qui se font une guerre par surimpression dans le générique de début de la série.

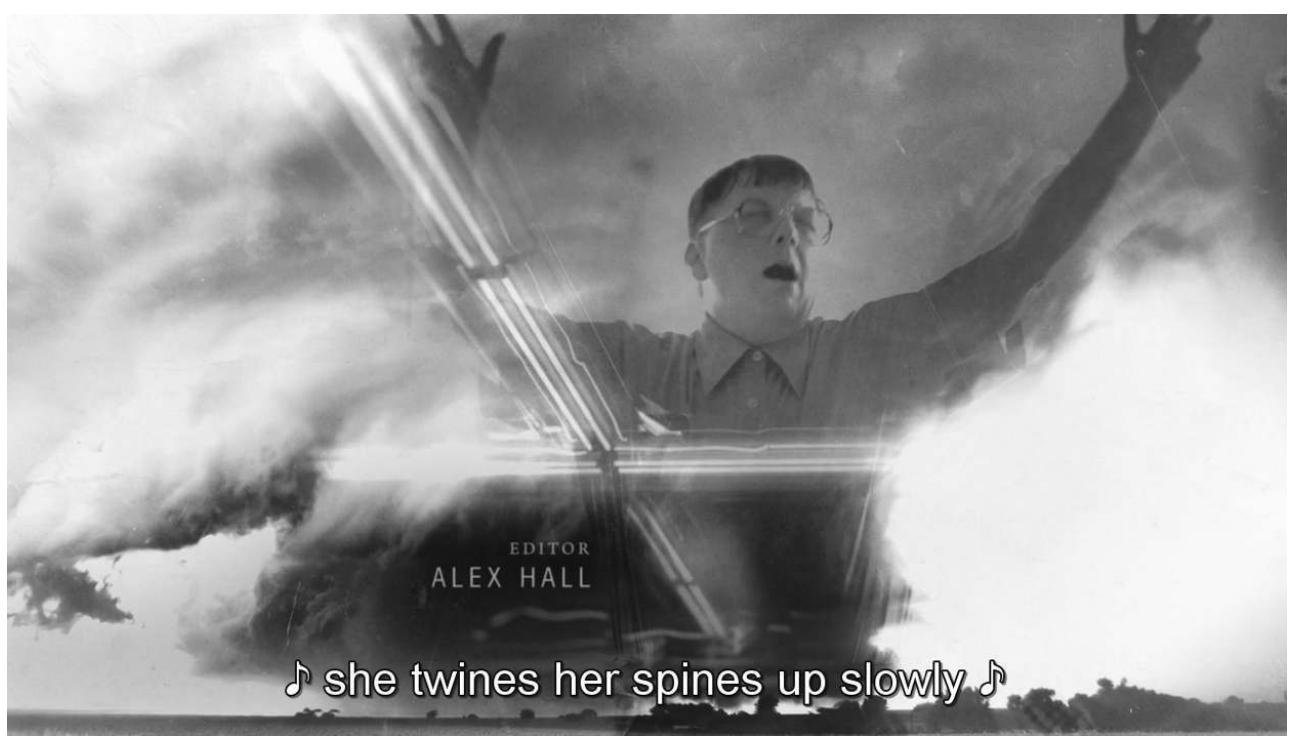

Fig 10 : Générique de la série 1 


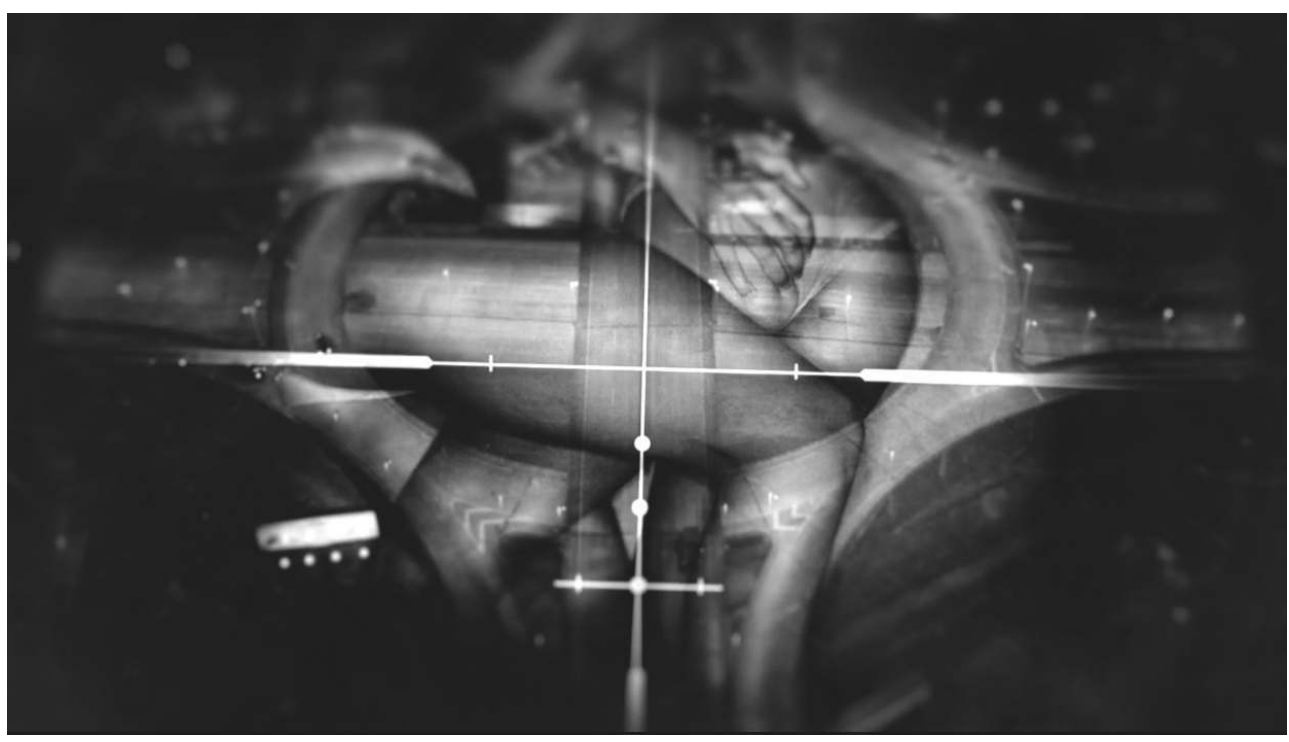

Fig 11 : Générique de la série 2

La confrontation de la nature féminine, sauvage et désordonnée et de l'ordre masculin et géométrique se manifeste plusieurs fois au travers de l'image récurrente d'hommes tondant la pelouse. Lorsque Rustin tond la pelouse de Martin à sa place, cela met ce dernier hors de lui. Et pour cause : la figure de l'homme tondant la pelouse est à la fois une représentation clichée du bon père de famille, une métaphore sexuelle douteuse et la façon par laquelle un homme, en mettant d'ailleurs en valeur comme le fait Cohle ses attributs masculins - une certaine muscularité rehaussée par sa sueur - ramène à l'horizontal la féminité tordue et sauvage de la « mauvaise herbe » pour la re-géométriser à son image. Tondre la pelouse est donc une marque de pouvoir viril qui transparaît par les lignes.

Mais un homme en particulier dans cette série a pour métier de tondre la pelouse : c'est le criminel Childress, que les détectives rencontrent par deux fois, assis sur sa tondeuse. Il va pourtant de soi dans la série que Childress ne tond pas réellement la pelouse mais en donne le spectacle, moquant et humiliant symboliquement par ce geste les détectives dont il se joue sans cesse. Son geste n'est que pantomime, que ce soit d'un point de vue pragmatique - la pelouse qu'il tond est systématiquement déjà tondue - ou symbolique - il tond devant les églises, dont on a vu qu'elles représentaient déjà des espaces parfaitement géométrisés, et dans des cimetières, comme s'il tondait les morts, horizontalisait l'horizontal. Par son action tautologique il prouve une fois pour toute qu'il est entièrement hors de ce combat dichotomique entre l'homme et la nature. Il est autre chose.

\section{Conclusion : Childress}

17 Childress est en effet dans le film une figure médiane, proprement monstrueuse puisqu'il pervertit toutes les notions prédéfinies de virilité, de sexualité et de famille. Marqué du signe de la spirale mais travaillant pour des Églises, défini comme le " Tall Man », figure virile et verticale, mais systématiquement décrit par sa large cicatrice aux formes courbes et retorses, il pervertit aussi bien les attributs de la Nature que ceux de la virilité. Entité qui défie les lois de la Nature et celles des hommes, de l'Etat, il se révèle dans la figure 
presque mythologique du "violeur d'enfants ", véritable épouvantail américain : le " Tall Man » est un Boogie-Man ${ }^{9}$ incarné. L'utilisation de masques d'animaux, les mises en scènes rituelles et les liens morbides et incestueux qu'il entretient avec sa famille ne sont pas sans faire penser à Leatherface, autre monstre enfermé dans un état de perversion de la Nature dans le film Chainsaw Massacre (Massacre à la tronçonneuse). Son nom même de Childress, qui à une lettre près signifie "sans enfants" (childless), met en valeur son rapport particulier à l'enfance, puisqu'il semble y être encore (sa stature de gros bébé et son rapport à son père en sont des indices parmi d'autres), mais fait aussi torturer, violer et assassiner des enfants. Par ailleurs sa famille a apparemment une longue tradition de perversion sexuelle, puisqu'il revit avec sa sœur la sexualité qu'elle avait elle-même avec leur grand-père lorsqu'il la violait.

18 Aussi, à la fois enfant et adulte, du côté de la virilité et de celle de la sauvagerie, grand mais tordu, Childress est le pivot autour duquel les détectives tournent en spirale aussi bien au niveau de leur enquête, de la narration globale de la série faite d'avants et d'après, qu'au niveau même de cet univers entièrement dichotomique que nous présente True Detective. Pacôme Thiellement et Thomas Bertay dans le premier volet de leur tétralogie Stupor Mundi ${ }^{10}$ proposent que le point de jonction ultime, celui qui est « au cœur de l'espace et du temps» (qu'ils appellent non sans mysticisme «la Grande Note ») ne peut être compris que par l'humain qui abandonne son humanité, sa normalité, pour revenir à un état transcendant, un état carnavalesque. Seuls les Freaks, les monstres, entendent la Grande Note et le Carnaval n'a pas d'autre objet que de nous transformer en monstre pour que cette réalité nous soit révélée. La lecture qu'a Nick Pizzolatto du monde qu'il crée n'est pas différente: c'est Childress, un monstre, qui est au centre de toutes choses, et c'est en devenant des monstres par des rituels de déguisement très carnavalesques que ceux qui lui gravitent autour tentent d'atteindre ce savoir premier.

La réconciliation de l'homme avec la nature n'est donc pour Pizzolatto possible que dans la monstruosité, mais aussi l'amoralité. L'espoir n'est pas ici dans la réconciliation mais dans la résignation. En quelque sorte, le seul espoir de victoire pour Rustin Cohle et Martin Hart est que leur virilité soit vaincue.

Les deux détectives finissent par se confronter réellement à ce pivot de perversion, notamment en pénétrant une forêt dans laquelle ni l'homme ni la nature n'ont réellement leur place: les bois sont brisés et les êtres à l'état de squelette. Mais ils échouent en partie contre le monstre et abandonnent dans le combat une part de leur virilité : tous deux sont pénétrés par les lames de Childress, allongés pour la première fois (leurs nuits d'insomnie ne leur permettant pas jusque là de quitter leur position verticale) et, par la suite, tous deux à tour de rôle éclateront en sanglots. 


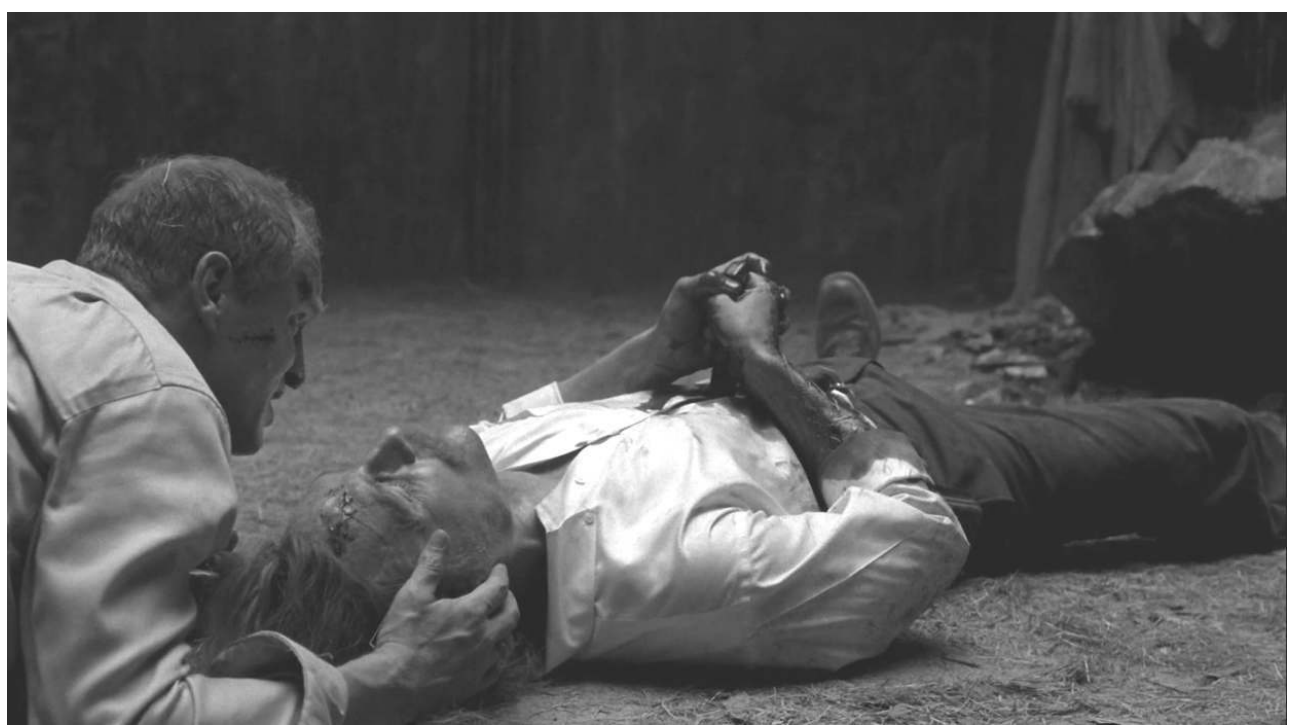

Fig 12 : Les détectives abandonnent une part de leur virilité

21 C'est cette perte de virilité qui leur permettra enfin de créer le lien tant recherché avec leur famille: Martin lorsque sa femme et ses deux filles viendront lui rendre visite à l'hôpital, et Rustin par les retrouvailles mystiques avec sa fille et son père dans son expérience de mort imminente. En allant au bout de leur virilité jusqu'au point où elle rejoint la perversion, ils la perdent et l'abandonnent pour renouer avec leur généalogie, et leur nature.

\section{NOTES}

1. «Passé un certain âge, un homme qui n'a pas de famille c'est une mauvaise chose »

2. «Je voulais tous les membres de cette famille que je pourrais trouver. Il n'y en avait pas. Vous savez à quel point c'est rare en Louisiane?»

3. « Nous sommes des créatures qui, selon les lois de la Nature, ne devraient pas exister »

4. «Je pense que la meilleure chose que nous et toute notre espèce pouvons faire est de refuser de donner libre cours à notre instinct, d'arrêter de nous reproduire et de marcher main dans la main vers notre propre extinction. »

5. «Elle m'a épargné du pêché d'être père »

6. « Le monde a besoin d'hommes mauvais. On tient les autres hommes mauvais à carreau. »

7. «Femme du genre de Donna Reed»

8. Le mont Driskill dans Bienville Parish (163 mètres)

9. Croque-mitaine américain

10. Stupor Mundi, Pacôme Thiellement et Thomas Bertay. Tétralogie en cours de réalisation dont seuls les deux premiers volets ont été diffusés. 


\section{AUTEUR}

\section{MARIE MAILLOS}

Marie Maillos est diplômée d'un Master Professionnel en Décor de cinéma à l'École Supérieure d'Audio-Visuel de Toulouse. Après avoir poursuivie des études de scénario aux États-Unis (SVA, New York) et en France (ESAV, Toulouse) elle obtient un Master Professionnel de scénario. Elle travaille depuis 2013 sur une thèse intitulée «Créateurs et créatures de séries télévisées : une poïétique du personnage » en tant que Doctorante Contractuelle au Laboratoire LARA-SEPPIA de l'UT2J. 